\title{
NOTE ON THE NORMAL PLANES TO A SURFACE IN A SPACE OF FOUR DIMENSIONS
}

\author{
By C. L. E. Moore
}

1. Introduction. In this note two questions are considered. First, under what conditions will the normal planes to a surface $F$ in a space of four dimensions envelop a second surface $F^{\prime}$. Second, under what conditions does a surface in this space have a surface parallel to it.

The normal planes to a surface in 4-space do not, in general, have an envelope. The locus of the points of intersection of one of these planes with the nearby planes is, in general, a nondegenerate conic while the loctus of the points of intersection of a tangent plane with the nearby tangent planes degenerates into two straight lines. So we have at once a necessary condition that the normal planes to a surface $F$ envelope a surface $F^{\prime}$, viz., the locus of the intersection of a given plane with the nearby planes factors into two straight lines. We shall see that this condition is also sufficient. In case this condition is identically satisfied the correspondence between $F$ and $F^{\prime}$ is such that the characteristics on $F$ (curves such that tangent planes at two nearby points intersect in a line) correspond to characteristics on $F^{\prime}$.

Two parallel surfaces in 3-space have the same congruence of normals and the distances between corresponding points is constant. The second question discussed in this paper is whether it is possible to have parallel surfaces in 4-space satisfying both of these conditions, and how many. It is found that, in general, there is no surface parallel to a given surface. In case certain relations are satisfied there is a two-parameter family of surfaces having the same congruence of normal planes, but the second condition of parallelism is not satisfied. However if the surface is such that the derivative of any normal vector has no component in the normal plane then there is a two-parameter family satisfying both conditions for parallelism. 
2. The intersection of nearby planes of a congruence. A normal plane to a surface is cut by the nearby normal planes in a conic. ${ }^{1}$ This is true for any congruence of planes. ${ }^{2}$

Let the family of planes, in homogeneous coördinates, be

$$
\Sigma A_{i} x_{i}=0, \quad \Sigma B_{i} x_{i}=0, \quad i=1,2 \ldots 5,
$$

where $A_{i}$ and $B_{i}$ are functions of two parameters $u, v$. The point of intersection of any plane with a nearby plane is given by the solutions of the equations

$$
\begin{aligned}
& \Sigma A_{i} x_{i}=0, \quad \Sigma B_{i} x_{i}=0, \\
& \Sigma\left(\frac{\partial A_{i}}{\partial u} d u+\frac{\partial A_{i}}{\partial v} d v\right) x_{i}=0, \\
& \Sigma\left(\frac{\partial B_{i}}{\partial u} d u+\frac{\partial B_{i}}{\partial v} d v\right) x_{i}=0 .
\end{aligned}
$$

The coördinates of the point of intersection will then be a quadratic expression in $d u: d v$ and consequently the locus of the point of intersection will be a conic. To determine the conditions under which the planes of (1) will be the tangent planes to a surface we will take the dual of this system of planes. By the duality the planes of the congruence will go into the two-parameter family of lines of a ruled hypersurface, the points of intersection of nearby planes will go into the 3 -spaces containing two nearby lines; that is, into the tangent hyperplanes to the ruled hypersurface. If the planes of (1) envelope a surface then the hypersurface will be such that it has only a two-parameter family of tangent hyperplanes each of which is tangent at every point of one of the generators of the hypersurface. ${ }^{3}$ If this is the case Segre showed that each line is cut by two nearby lines. By the duality two lines that cut correspond to two planes of (1) which lie in the same 3 -space or which have a line in common. Hence if the planes of (1) are the tangent planes of a surface it is necessary

1 Wilson and Moore, Differential geometry of two dimensional surfaces in hyperspace, Proc. Amer. Acad. Arts and Sciences, vol. 52, 1916, p. 326.

2 Segre, Preliminari di una theoria delle varieat luoghi di spazi, Rend. Circolo Math. di Palermo, vol. xxx, 1910, pp. 87-121.

3 Segre, loc. cit. 
and sufficient that the conic (the locus of the points in which a given plane is cut by the nearby planes) should degenerate into a pair of straight lines. This relation of course is to be identically satisfied.

If (1) is the congruence of normal planes to a surface then the locus of intersection of a given plane with the nearby planes will be the inverse of the pedal of the indicatrix." If the normal planes envelope a surface this locus will be two straight lines. The pedal of the indicatrix will then become two circles passing through the surface point, which means that the tangents to the indicatrix will have to pass through two points and hence the indicatrix (which is always an ellipse the points of which correspond to real directions on the surface) will degenerate into a line segment. The necessary and sufficient condition that the indicatrix reduce to a line segment (not passing through the surface point) is that the characteristics (directions on the surface such that the tangent planes at two nearby points will intersect in a line) intersect at right angles. Hence if the characteristics of a surface $\mathrm{F}$ form an orthogonal curve system on the surface, the normal planes to the surface will envelop a second surface $\mathrm{F}^{\prime}$.

The correspondence between $F$ and $F^{\prime}$ is such that the characteristics of $F$ correspond to the characteristics of $F^{\prime}$. For if the indicatrix of $F$ is a line segment then the characteristics of $F$ correspond to the ends of this segment. The tangents to the characteristics of $F^{\prime}$ will be the lines into which the locus of the intersection with nearby planes degenerates, but these two lines are seen to correspond to the ends of the indicatrix of $F$. If this indicatrix is $A B$ then the characteristic corresponding to $A$ is such that tangent planes at nearby points in this direction intersect in a line tangent to the other characteristic ${ }^{5}$ and likewise for $F^{\prime}$. Hence the characteristic of $F$ corresponding to $A$ will correspond to the characteristic of $F^{\prime}$ which corresponds to $B$.

If $F$ is a developable whose indicatrix reduces to a line segment then the lines joining the ends of the indicatrix to the surface point intersect at right angles ${ }^{6}$ and hence the characteristics of

4 Wilson Moore, loc. cit., p. 328.

5 Segre, Su una clarse di superficie degl' iperspazii, Atti Accad. Realle delle Scienze di Torino, 1906-7.

6 Wilson and Moore, loc. cit., p. 332. 
$F^{\prime}$ will be orthogonal and consequently the normal planes of $F^{\prime}$ will also envelop a surface $F^{\prime \prime}$. An example of such a surface is the surface generated by rotating a logarithmic spiral in the $x_{1} x_{3}=$ plane by the rotation ${ }^{7}$

$$
\begin{array}{ll}
x_{1}{ }^{\prime}=x_{1} \cos t-x_{2} \sin t, & x_{2}{ }^{\prime}=x_{1} \sin t+x_{2} \cos t, \\
x_{3}{ }^{\prime}=x_{3} \cos t-x_{4} \sin t, & x_{4}{ }^{\prime}=x_{3} \sin t+x_{4} \cos t .
\end{array}
$$

3. Parallel surfaces. In order to determine the condition for parallel surfaces we will investigate the condition that a congruence of planes have a surface normal to them; that is, the condition that the given congruence be the congruence of normal planes of a surface. Let the given congruence be represented vectorially by

$$
\xi=a+b t+c \tau,
$$

where $a, b, c$ are vectors, functions of $u, v$, and $t$ and $\tau$ are parameters. When $t$ and $\tau$ vary while $u, v$ are held fixed $\xi$, will generate the given plane of the congruence. We will assume that $b$ and $c$ satisfy the relations

$$
b^{2}=c^{2}=1, \quad b . c=0 .
$$

From (4) by differentiation we have

$$
b . d b=c . d c=0, \quad b . d c+d b . c=0 .
$$

The necessary and sufficient condition that (3) be a congruence of normal planes is that it is possible to determine $t$ and $\tau$ as functions of $u, v$, so that the resulting surface has $\xi$ for its normal plane. If this is possible then $d \xi$ will be normal to both $b$ and $c$ since $b$ and $c$ are two vectors lying in the plane of the congruence. Then

$$
d \xi . b=d \xi . c=0 .
$$

Which, using (5), reduces to

$$
\begin{aligned}
& b . d a+d t+\tau b . d c=0, \\
& c . d a+d \tau+t c . d b=\mathbf{0} .
\end{aligned}
$$

7.C. L. E. Moore, Surfaces of rotation in a space of four dimensions, Annals of Math., vol. xxi, 1919. 
Expanding in terms of $d u, d v$, and putting in the condition that $d u, d v$ are arbitrary, we have

$$
\begin{aligned}
& b \cdot \frac{\partial a}{\partial u}+\tau b \cdot \frac{\partial c}{\partial u}=+\frac{\partial t}{\partial u}, \\
& b \cdot \frac{\partial a}{\partial v}+\tau b \cdot \frac{\partial c}{\partial v}=-\frac{\partial t}{\partial v}, \\
& c \cdot \frac{\partial a}{\partial u}+t c \cdot \frac{\partial b}{\partial u}=-\frac{\partial \tau}{\partial u}, \\
& c \cdot \frac{\partial a}{\partial v}+t c \cdot \frac{\partial b}{\partial v}=-\frac{\partial \tau}{\partial v} .
\end{aligned}
$$

Putting

$$
\begin{gathered}
b \cdot \frac{\partial a}{\partial u}=-A, \quad b \cdot \frac{\partial a}{\partial v}=-B, \quad c \cdot \frac{\partial a}{\partial u}=-C, \quad c \cdot \frac{\partial a}{\partial v}=-D, \\
b \cdot \frac{\partial c}{\partial u}=-c \cdot \frac{\partial b}{\partial u}=-P, \quad b \cdot \frac{\partial c}{\partial v}=-c \cdot \frac{\partial b}{\partial v}=-Q,
\end{gathered}
$$

equations (6) become

$$
\begin{array}{ll}
A+P \tau=\frac{\partial t}{\partial u}, & B+Q \tau=\frac{\partial t}{\partial v}, \\
C-P t=\frac{\partial \tau}{\partial u}, & D-Q t=\frac{\partial \tau}{\partial v} .
\end{array}
$$

We are then to determine the solutions of this set of equations in terms of $u, v$. It is obvious that this set will not be consistent unless

$$
\begin{aligned}
& \frac{\partial}{\partial v}\left(A+P \tau=\frac{\partial}{\partial u}(B+Q \tau),\right. \\
& \frac{\partial}{\partial v}(C-P t)=\frac{\partial}{\partial u}(D-Q t) .
\end{aligned}
$$

Expanding these and substituting the values of $\frac{\partial t}{\partial u}, \frac{\partial t}{\partial v}, \frac{\partial \tau}{\partial u}, \frac{\partial \tau}{\partial v}$, 
from (7) we obtain

$$
\begin{gathered}
\tau\left(\frac{\partial P}{\partial v}-\frac{\partial Q}{\partial u}\right)=\frac{\partial B}{\partial u}-\frac{\partial A}{\partial v}+Q C-P D \\
t\left(\frac{\partial P}{\partial v}-\frac{\partial Q}{\partial u}\right)=\frac{\partial C}{\partial v}-\frac{\partial D}{\partial u}+Q A-P B
\end{gathered}
$$

The values of $t$ and $\tau$ are therefore, in general, uniquely determined. The values of $t$ and $\tau$ from (8) will obviously not satisfy (7) unless certain relations are satisfied by the coefficients. These relations are obtained by direct substitution in (7). A set of normal planes to a surface will satisfy these conditions, but this shows that, in general, there is no other surface having the same set of normal planes.

If

$$
\frac{\partial P}{\partial v}-\frac{\partial Q}{\partial u}=0,
$$

then $t, \tau$ cannot be determined from (8). In this case, in addition to $(9)$, the coefficients will satisfy the relations

$$
\begin{aligned}
& \frac{\partial B}{\partial u}-\frac{\partial A}{\partial v}+Q C-P D=0, \\
& \frac{\partial C}{\partial v}-\frac{\partial D}{\partial u}+Q A-P B=0,
\end{aligned}
$$

and we have to solve (7) subject to relations (9) and (10). We can evidently make the coefficients satisfy enough relations besides these so that ( 7$)$ will have a solution. Suppose this done and let $t_{0}, \tau_{0}$ be any particular solution of (7), then the general solution can be expressed by

$$
t=t_{0}+t_{1}, \quad \tau=\tau_{0}+\tau_{1},
$$

where $t_{1}, \tau_{1}$ satisfy the reduced system

$$
\begin{array}{ll}
\frac{\partial t_{1}}{\partial u}=P \tau_{1}, & \frac{\partial t_{1}}{\partial v}=Q \tau_{1}, \\
\frac{\partial \tau_{1}}{\partial u}=-P t_{1}, & \frac{\partial \tau_{1}}{\partial v}=-Q t_{1} .
\end{array}
$$


Eliminating $\boldsymbol{\tau}_{1}$ from the first two,

$$
Q \frac{\partial t_{1}}{\partial u}-P \frac{\partial t_{1}}{\partial v}=0
$$

The solution of this last equation is an arbitrary function of the solution of

$$
P d u+Q d v=0 \text {, }
$$

which from $(9)$ is seen to be exact. Let $f(u, v)$ satisfy

$$
d f=P d u+Q d v,
$$

then the solution of (12) is

$$
t_{1}=F(f) .
$$

Substituting this in the first equation of (11),

$$
\tau_{1}=\frac{d F(f)}{d f}=F^{\prime}(f) .
$$

Putting these values in the last equation of (11)

$$
F^{\prime \prime}=-F \text {, }
$$

and hence

$$
\begin{aligned}
& t_{1}=K_{1} \cos \left(f+K_{2}\right), \\
& \tau=-K_{1} \sin \left(f+K_{2}\right) .
\end{aligned}
$$

These values satisfy (11). We can then write the general solution of $(7)$ in the form

$$
\begin{aligned}
& t=t_{0}+K_{1} \cos \left(f+K_{2}\right), \\
& \tau=\tau_{0}-K_{1} \sin \left(f+K_{2}\right) .
\end{aligned}
$$

In this case, then, there is a two-parameter family of surfaces having the same congruence of normal planes, but obviously the distance between corresponding points is not constant and consequently the surfaces are not truly parallel.

If

$$
P=Q=0 \text {, }
$$

relation (10) reduces to

$$
\frac{\partial B}{\partial u}-\frac{\partial A}{\partial v}=0, \quad \frac{\partial D}{\partial u}-\frac{\partial C}{\partial v}=0,
$$


and the solutions of the equations (7) are then the solutions of the two exact equations,

$$
\begin{aligned}
& A d u+B d v=d t \\
& C d u+D d v=d \tau,
\end{aligned}
$$

or

$$
t=t_{0}+K_{1}, \quad \tau=\tau_{0}+K_{2} .
$$

From (3) if $K_{1}=K_{2}=0$, the surface point is determined by putting in the values of $t_{0}, \tau_{0}$. Then for values of $K_{1}$ and $K_{2}$ different from zero we see that the distance between corresponding points is

$$
K_{1} b+K_{2} c
$$

and since $b, c$ are unit vectors this is the same for all corresponding points. Hence in this case there is a two-parameter family of parallel surfaces. This is the real generalization of parallel surfaces in 3-space. An example of a surface of this kind is the surface generated by rotating a circle with center at the origin and lying in the $x_{1} x_{3}$-plane by the rotation (2). This is a developable surface and all the normal planes pass through $O$.

If (3) represents the normal planes to a surface then by the notation used by Wilson and Moore ${ }^{\mathbf{s}} b=z, c=w$, and the relation $P=Q=0$ is

$$
z . w_{s}=v_{s}=0 \text {. }
$$

Hence for this last case $\nu_{s}=0$; hence $\nu_{s}=0$ is the necessary and sufficient condition that the surface be one of a two-parameter family of parallel surfaces, or the necessary and sufficient condition that a surface belong to a family of parallel surfaces is that the derivatives of the normals to the surface have no component in the normal. plane.

From the fact that the locus of intersection of nearby normal planes is the inverse of the pedal of the indicatrix it follows that the character of the indicatrix will be the same for all surfaces having the same congruence of normal planes. That is, if the indicatrix of one reduces to a line segment, all the others will have the same property; if the indicatrix of one passes through the surface point, so will it for all the others, etc.

8 Loc. cit., p. 304. 
4. Families of planes of $n-2$ parameters in $n$-space. The results of the last section can readily be extended to space of $n$ dimensions. Let the family of planes be represented vectorially by

$$
\xi=a+b t+c \tau,
$$

where $a, b, c$ are vectors, functions of $u_{1}, u_{2} \ldots n_{n-2}$ and $b, c$ satisfy the relations

$$
b^{2}=c^{2}=1, \quad b . c=0 .
$$

The condition, then, that a variety $v_{n-2}$ have these planes for normal planes is the same as before and by similar notation is

$$
\begin{aligned}
A_{i}+P_{i} \tau & =\frac{\partial t}{\partial u_{i}}, \quad i=1,2, \ldots n-2 . \\
B_{i}-P_{i} t & =\frac{\partial \tau}{\partial u_{i}} .
\end{aligned}
$$

Putting in the condition for exact differentials as before, we obtain equations exactly similar to (8). Hence, in general, there is a single $v_{n-2}$ normal to a normal family of planes. In case $\Sigma P_{i} d u_{i}$ is an exact differential, there is a two-parameter family of such varieties. For, as before, the solution of the reduced system

$$
\Sigma P_{i} \tau=\frac{\partial t}{\partial u_{i}}, \quad-\Sigma P_{i} t=\frac{\partial \tau}{\partial u_{i}},
$$

is

$$
t=K_{1} \cos \left(f+K_{2}\right), \quad \tau=-K_{1}, \sin \left(f+K_{2}\right),
$$

and the solution of the general set is

$$
t=t_{0}+K_{1} \cos \left(f+K_{2}\right), \quad \tau=\tau_{0}-K_{1} \sin \left(f+K_{2}\right),
$$

where $f$ satisfied $d f=\Sigma P_{i} d u_{i}$. The varieties in this case are not parallel, but if

$$
P_{i}=0
$$

then there is a two-parameter family of parallel varieties having the same family of normal planes. 
5. The general case. The condition that

$$
\xi=a_{i}+a_{i} t_{i}, \quad i=1,2, \ldots K,
$$

$a_{i}, a_{i}$ being functions of $n-k$ parameters $u_{j}$, be the normal spaces to a variety $v_{n-k}$, by similar notation and assumptions on $a_{i}$ can be written

$$
A_{i j}+\underset{k}{\Sigma} P_{k i j} t_{k}=\frac{\partial t_{i}}{\partial u_{j}}
$$

The coefficients satisfy the following relations:

$$
A_{i j}=A_{j i}, \quad P_{i i j}=0, \quad P_{k i j}=-P_{i k j} .
$$

Putting in the condition for an exact differential we are led to the statement that, in general, when $\xi$ is a normal system there is only variety $v_{n-k}$ having it for its system of normals, but that there are cases when there is an infinite system of such varieties but that these are not parallel except in the case

$$
P_{k i j}=0 \text {. }
$$

There is an infinite set of parallel varieties (if $\left.\frac{\partial A_{i k}}{\partial u_{j}}=\frac{\partial A_{i j}}{\partial u_{k}}\right)$. 